\title{
Impact of Rice Straw Mulch on Growth, Yield Components and Yield of Direct Seeded Lowland Rice (Oryza sativa L.)
}

\author{
D.A.U.D. Devasinghe. K.P. Premaratne ${ }^{1 *}$ and U.R. Sangakkara ${ }^{1}$ \\ Department of Plant Sciences, Faculty of Agriculture \\ Rajarata University of Sri Lanka \\ Puliyankulama, Anuradhapura \\ Sri Lanka
}

\begin{abstract}
The impact of a rice straw mulch (RSM) under optimal mineral fertilized conditions on growth, yield components and yield of dry-direct seeded (DDSR) and wetdirect seeded rice (WDSR) was evaluated under field conditions over four consecutive seasons to include two dry (DS) and two wet (WS) seasons, in the dry zone of Sri Lanka. The RSM could be applied in WDSR in both seasons and DDSR in WS at a rate of 4t/ha without affecting the seedling density. RSM in WDSR, especially at initial stages of plant growth (i.e. 35 days after sowing) increased plant height, length of the longest root and total root length significantly than in non mulched and DDSR-RSM plots. Shoot and root biomass were also significantly increased by RSM in WDSR in both seasons. Rice straw mulch increased panicle number per unit area and number of spikelets per panicle in WDSR-RSM in both seasons. A significantly high yield could be achieved in WDSR-RSM, WDSR-chemical weeding $(C)$ and DDSR-C treatments in both seasons. The yield enhancement was attributed to yield components, principally panicle number per unit area and number of spikelet per panicle. There was a greater panicle number per unit area in the DS while the WS favors more spikelets per panicle. The study indicated the possibility of enhancing the rice plant growth, yield components and yields of rice in wet- direct seeded lowland rice by rice straw mulch in both seasons in this region.
\end{abstract}

Key words: Dry-direct seeded rice, grain, mulch, wet- direct seeded rice, yield components

\section{INTRODUCTION}

Nutrient cycling in the ecosystem is an essential component of sustainable rice production. Although during the last three decades mineral fertilization played a dominant role in the rice-based cropping systems, in recent years, there have been serious concerns about longterm adverse effects of continuous and indiscriminate use of inorganic fertilizers on soil structure, soil health and environmental pollution (Singh, 2000). In contrast, crop residues, the harvest remnants of the previous crop, have been identified as an essential component in the cycling of nutrients in rice ecosystems (Singh et al., 2005).

Tropical agricultural ecosystems are affected by rapid biological degradation of soils, which results in the reduction of soil organic matter due to the decline in $\mathrm{C}$ inputs from biomass (Stewart and Robinson, 1997). In tropical systems, mineralization rates are potentially higher than in temperate ones because of high soil temperatures during cropping seasons,

\footnotetext{
${ }^{1}$ Department of Crop Science, Faculty of Agriculture, University of Peradeniya, Peradeniya, Sri Lanka

* Author of Correspondent: kp.premaratne@gmai.com
} 
particularly at the time of incorporation of residues (Singh et al., 2005). Tropical soils also vary widely in their properties and are generally poor in inherent soil fertility to support high crop productivity. The removal of crop residues lowers soil fertility and thereby decreases crop production (Singh et al., 2005). Thus, incorporation of organic materials such as crop residues offers a sustainable and ecologically sound alternative for meeting the nutrient requirements of crops (Boyle et al., 1989).

Significant quantities of rice residues are produced in the rice growing countries. Moreover, the adoption of mechanized farming has resulted in leaving a significant amount of rice straw in the field after harvesting the grain. Thus, there is an enormous potential of recycling these residues in the crop production systems (Mandal et al., 2004). As indicated by Mendoza and Samson (1999) the use of rice straw mulching for weed control in different crops is possible. Hence, weed control coupled with yield enhancements by rice straw mulching would be beneficial in integrated plant management systems, while minimizing the impact of agrochemicals; which is an important concern in current agricultural activities.

This study was carried out to investigate the effect rice straw as mulch on growth, yield components and yield of wet- direct seeded (broadcasting of pre-germinated seeds in to puddle soil) and dry- direct seeded (broadcasting of non-germinated seeds in to dry/moist soil) lowland rice over the two principal rice growing seasons in the dry zone of Sri Lanka over four consecutive seasons.

\section{MATERIALS AND METHODS}

\section{Experimental site and treatments of study}

This experiment was conducted over two years; covering four seasons to encompass two dry (DS; 2009 and 2010) and two wet seasons (WS; 2009/10 and 2010/11) at the research unit of the Faculty of Agriculture, Rajarata University of Sri Lanka. Soil pH, total N, available- P, and extractable- K were $7.20 \pm 0.21,0.28 \pm 0.004 \%, 10.00 \pm 0.81 \mathrm{ppm}$ and $62.00 \pm 0.47 \mathrm{ppm}$ respectively prior to crop establishment at the experimental site. The rainfall during the study period in 2009 DS, 2009/10 WS, 2010 DS and 2010/11 WS was $72.5 \mathrm{~mm}, 650.4 \mathrm{~mm}, 209.8$ $\mathrm{mm}$ and $1386.7 \mathrm{~mm}$ respectively. The mean seasonal temperatures were $29.00 \pm 0.34{ }^{\circ} \mathrm{C}$ and $26.00 \pm 1{ }^{\circ} \mathrm{C}$ for DS and WS respectively.

The treatments were evaluated using a randomized complete block design which included the rice establishment method and weed management. The treatment combinations were drydirect seeded rice (DDSR) - non-weeded (UW), DDSR- Rice straw mulch (RSM), DDSRChemical weeding (C), wet- direct seeded rice (WDSR)-UW, WDSR-RSM and WDSR-C. These six treatments were repeated in three adjacent blocks thus having a total of eighteen plots with dimensions of $6 \times 3 \mathrm{~m}$.

\section{Crop management}

Tillage operations for DDSR and WDSR were practiced separately, prior to the initiation of intense monsoon rains in each season. First, nine plots where wet- direct seeding was practiced were tilled two weeks prior to crop establishment and water was impounded. The harrowing was done after two weeks in those plots followed by fine leveling. At the same date, in other nine plots where dry- direct seeding was practiced, the soil was moistened to field capacity followed by ploughing, harrowing and rough leveling. 
An improved rice variety $\mathrm{Bg} 352$ (duration 3.5 months) was used. WDSR plots were broadcast sown with pre-germinated seeds into puddled soil at a seed rate of $137.5 \mathrm{~kg} / \mathrm{ha}$. DDSR plots were sown with dry, non-germinated seeds on to moist plots at the same rate. In six plots, where the rice straw was a treatment, a layer of air dried rice straw collected from the previous rice crop of $\mathrm{Bg} 352$ was applied uniformly, just after sowing at the rate of $4 \mathrm{t} / \mathrm{ha}$ (i.e. $400 \mathrm{~g} / \mathrm{m}^{2}$ ). The application of rice straw mulch at this rate formed a $2 \mathrm{~cm}$ thick straw layer on the soil surface. Another six plots where the chemical weeding was a treatment, Ethoxysulfuron $20+$ fenoxaprop $69 \mathrm{~g}$ a.i./L (Tiller ${ }^{\circledR}$ Gold) was applied at a rate of $0.5 \mathrm{~L} / \mathrm{ha}$ between 10-14 days after sowing.

Identical NPK fertilizer rates (120 - 40 - $40 \mathrm{~kg}$ of N, $\mathrm{P}_{2} \mathrm{O}_{5}$ and $\mathrm{K}_{2} \mathrm{O}$ per ha respectively) were used as recommended by the Department of Agriculture in Sri Lanka (DOA, 2001) for all treatments. All the $\mathrm{P}_{2} \mathrm{O}_{5}, 20 \mathrm{~kg} / \mathrm{ha}$ of $\mathrm{K}_{2} \mathrm{O}$ and $5 \mathrm{~kg} / \mathrm{ha}$ of $\mathrm{N}$ were applied at the time of crop establishment. The remaining $\mathrm{N}$ was applied at 14 days after sowing ( $35 \mathrm{~kg} / \mathrm{ha}), 35$ days after sowing $(55 \mathrm{~kg} / \mathrm{ha})$ and at panicle initiation stage $(25 \mathrm{~kg} / \mathrm{ha})$. The balance $\mathrm{K}_{2} \mathrm{O}(20 \mathrm{~kg} / \mathrm{ha})$ was applied at panicle initiation stage of the rice crop. After establishment of the rice crop, all plots were irrigated at 5 day intervals and water level was always kept at 2-3 cm above the soil surface at the time of irrigation, until crop maturity.

\section{Measurements}

Rice plants were sampled at 14 days after sowing for seedling density using four randomly selected quadrats of $0.5 \mathrm{~m} \times 0.5 \mathrm{~m}$ from each plot. Plant height, length of the longest root, total plant root length in rice was measured at 14, 28, 35 days after sowing (DAS), panicle initiation, and 50\% heading stages. The plant height was measured using a scaled ruler from base of the culm to growing point of the plant. The length of the longest root was measured from the base of the culm to the tip of the longest root. The plant root length was recorded as an averaged value of cumulative sums of all roots of randomly selected twenty plants from each plot. At 28, 35 DAS, panicle initiation, and 50\% heading and harvesting stages the rice plants were sampled for root and shoot biomass. To obtain the root and shoot biomass of the rice plant, the sampled rice plants were separated into roots and shoots and dried at $70{ }^{\circ} \mathrm{C}$ for $48 \mathrm{hrs}$ in an oven to a constant weight and weighed. At the time of harvesting the yield components (panicle number per unit area, number of spikelets per panicle, percentage of filled spikelets, and 1000- grain weight) were also recorded. In addition, the grain yield (adjusted to $14 \%$ moisture content) was determined at harvest using yield components as done by Yoshida (1981).

$\operatorname{Yield}\left(\mathrm{g} / \mathrm{m}^{2}\right)=\left(\begin{array}{l}\text { Panicle number } / \mathrm{m}^{2} \times \text { Spikeletsnumber/panicle } \times \% \text { Filled spikelets } \\ \times 1000-\text { grain weight }(\mathrm{g}) \times 10^{-5}\end{array}\right)$

\section{Data analysis}

The comparison of treatments was done on a seasonal basis (dry seasons and wet seasons). Prior to the detailed analysis, the data were tested for normality. Analysis of variance was carried out for the collected data using the SAS statistical analytical package (Version 8.12, SAS Institute, Cary, NC). The Duncan's test was used for mean separation procedure when the treatment effects were significant. 


\section{RESULTS}

\section{Seedling density of rice}

In the WS, a significantly higher $(\mathrm{p}=0.0002)$ seedling density was observed than in the DS (Table 1). The rice straw mulch with a thickness of $2 \mathrm{~cm}$ on the soil surface did not affect the emergence of rice seedlings in WDSR method in both seasons. In contrast, a significant reduction in seedling density was observed in DDSR-RSM in DS (Table 1). The season and treatment interaction was not significant $(\mathrm{p}=0.19)$ for seedling density.

Table 1. Seedling density of rice as affected by season, method of crop establishment and rice straw mulch

\begin{tabular}{|c|c|c|}
\hline Season & \multicolumn{2}{|c|}{ Seedling density (Seedlings/m²) } \\
\hline DS & \multicolumn{2}{|c|}{$495^{\mathrm{b}}$} \\
\hline WS & \multicolumn{2}{|c|}{$546^{\mathrm{a}}$} \\
\hline $\mathrm{CV} \%$ & \multicolumn{2}{|c|}{10.34} \\
\hline Treatment & DS & WS \\
\hline DDSR-UW & $508^{a}$ & $556^{\mathrm{a}}$ \\
\hline DDSR-RSM & $408^{b}$ & $529^{\mathrm{a}}$ \\
\hline DDSR-C & $521^{\mathrm{a}}$ & $544^{\mathrm{a}}$ \\
\hline WDSR-UW & $510^{a}$ & $540^{\mathrm{a}}$ \\
\hline WDSR-RSM & $520^{\mathrm{a}}$ & $556^{\mathrm{a}}$ \\
\hline WDSR-C & $501^{\mathrm{a}}$ & $526^{\mathrm{a}}$ \\
\hline CV \% & 14.21 & 4.76 \\
\hline
\end{tabular}

DDSR, dry- direct seeded rice; WDSR, wet- direct seeded rice; UW, non-weeding; C, chemical; RSM, rice straw mulch; DS, dry season; WS, Wet season

In a column, values followed by a common letter are not significantly different at $\alpha=0.05$

\section{Plant height}

The seasonal impact on plant height was significant at 28, 35 days after sowing and at panicle initiation $(\mathrm{P}=<0.0001)$ and significantly taller rice plants were seen in the WS. However, at 50\% heading stage, plant height was significantly higher in DS than in the WS (Table 2). The height of plants in WDSR was significantly increased in all sampling dates by the rice straw mulch, regardless the crop duration in both seasons (Table 2). Hence, at each sampling dates, the tallest plant was in WDSR- RSM treatment, when compared to the nonmulched and DDSR-RSM treatments in both seasons. At $50 \%$ heading stage, the rice plants in WDSR-C plots were similar to the height of WDSR-RSM in DS (Table 2). The increase in height was more between 47-67 days after sowing in all treatments of study, as it was the maximum vegetative stage of the rice plant.

\section{Length of the longest root and total root length}

The rice straw mulch affected root characteristics in terms of the length of the longest root and total plant root length. In both seasons, a significant increase in the length of the longest root (Table 3) and total plant root length (Table 4) was recorded in WDSR-RSM at all dates of sampling. Among non-mulched treatments the highest length of the longest root and total plant root length was observed in the WDSR-C treatment. Compared to WDSR-C, the enhancement of length of the longest root and total plant root length was greater in WDSR- 
RSM in both seasons. Hence, length of the longest root and total plant root length was increased due to rice straw mulching in WDSR method than in the present practice of WDSR-C was clearly evident.

Table 2. Plant height of rice as affected by season, method of crop establishment and weed management

\begin{tabular}{|c|c|c|c|c|c|c|c|c|c|c|}
\hline \multicolumn{11}{|c|}{ Plant height $(\mathbf{c m})$} \\
\hline Season & \multicolumn{2}{|c|}{14 DAS } & \multicolumn{2}{|c|}{ 28DAS } & \multicolumn{2}{|c|}{35 DAS } & \multicolumn{2}{|c|}{ PI } & \multicolumn{2}{|c|}{$50 \% \mathrm{H}$} \\
\hline DS & \multicolumn{2}{|c|}{$5.1^{\mathrm{a}}$} & \multicolumn{2}{|c|}{$7.7^{\mathrm{b}}$} & \multicolumn{2}{|c|}{$9.1^{b}$} & \multicolumn{2}{|c|}{$16.5^{\mathrm{b}}$} & \multicolumn{2}{|c|}{$47.5^{\mathrm{a}}$} \\
\hline WS & \multicolumn{2}{|c|}{$5.2^{\mathrm{a}}$} & \multicolumn{2}{|c|}{$9.9^{\mathrm{a}}$} & \multicolumn{2}{|c|}{$12.5^{\mathrm{a}}$} & \multicolumn{2}{|c|}{$18.3^{\mathrm{a}}$} & \multicolumn{2}{|c|}{$46.5^{\mathrm{b}}$} \\
\hline $\mathrm{CV} \%$ & \multicolumn{2}{|c|}{9.6} & \multicolumn{2}{|c|}{7.7} & \multicolumn{2}{|c|}{8.1} & \multicolumn{2}{|c|}{6.9} & \multicolumn{2}{|c|}{10.6} \\
\hline & \multicolumn{2}{|c|}{14 DAS } & \multicolumn{2}{|c|}{ 28DAS } & \multicolumn{2}{|c|}{35 DAS } & \multicolumn{2}{|c|}{ PI } & \multicolumn{2}{|c|}{$50 \% \mathrm{H}$} \\
\hline Trt. & DS & WS & DS & WS & DS & WS & DS & WS & DS & WS \\
\hline DDSR-UW & $4.8^{b}$ & $4.9^{\mathrm{bcd}}$ & $7.8^{b}$ & $10.0^{\mathrm{b}}$ & $9.1^{b}$ & $11.5^{\mathrm{c}}$ & $14.9^{\mathrm{c}}$ & $17.4^{\mathrm{b}}$ & $42.1^{\mathrm{b}}$ & $44.0^{b}$ \\
\hline DDSR-RSM & $5.0^{\mathrm{b}}$ & $4.7^{c}$ & $7.2^{b c}$ & $10.0^{\mathrm{b}}$ & $8.4^{\mathrm{b}}$ & $12.8^{\mathrm{b}}$ & $15.8^{\mathrm{c}}$ & $17.4^{\mathrm{b}}$ & $43.8^{b}$ & $46.4^{b}$ \\
\hline DDSR-C & $4.7^{\mathrm{b}}$ & $4.6^{\mathrm{d}}$ & $6.8^{\mathrm{c}}$ & $9.3^{\mathrm{bc}}$ & $8.3^{b}$ & $12.1^{\mathrm{bc}}$ & $14.8^{\mathrm{c}}$ & $17.6^{\mathrm{b}}$ & $42.2^{\mathrm{b}}$ & $44.9^{b}$ \\
\hline WDSR-UW & $5.0^{\mathrm{b}}$ & $5.2^{\mathrm{bc}}$ & $7.4^{\mathrm{bc}}$ & $8.9^{c}$ & $8.3^{b}$ & $11.8^{\mathrm{bc}}$ & $17.1^{\mathrm{b}}$ & $17.6^{\mathrm{b}}$ & $46.6^{\mathrm{b}}$ & $42.4^{\mathrm{b}}$ \\
\hline WDSR-RSM & $6.0^{\mathrm{a}}$ & $6.4^{\mathrm{a}}$ & $9.2^{\mathrm{a}}$ & $11.4^{\mathrm{a}}$ & $10.6^{\mathrm{a}}$ & $14.0^{\mathrm{a}}$ & $18.9^{\mathrm{a}}$ & $21.7^{\mathrm{a}}$ & $56.7^{\mathrm{a}}$ & $53.8^{\mathrm{a}}$ \\
\hline WDSR-C & $5.2^{\mathrm{b}}$ & $5.3^{b}$ & $7.6^{\mathrm{b}}$ & $9.8^{\mathrm{b}}$ & $9.4^{\mathrm{b}}$ & $12.5^{\mathrm{bc}}$ & $17.3^{\mathrm{b}}$ & $17.8^{\mathrm{b}}$ & $53.8^{\mathrm{a}}$ & $46.8^{\mathrm{b}}$ \\
\hline $\mathrm{CV} \%$ & 10.4 & 8.1 & 7.8 & 7.4 & 9.7 & 7.19 & 5.9 & 7.1 & 10.8 & 8.7 \\
\hline
\end{tabular}

DDSR, dry- direct seeded rice; WDSR, wet- direct seeded rice; UW, non- weeding; C, chemical; RSM, rice straw mulch; DS, dry season: WS, Wet season; DAS, days after sowing; Trt, treatment; PI, panicle initiation; H, heading In a column, values followed by a common letter are not significantly different at $\alpha=0.05$

Table 3. Length of the longest root of rice as affected by season, method of crop establishment and weed management

\begin{tabular}{|c|c|c|c|c|c|c|c|c|c|c|}
\hline \multicolumn{11}{|c|}{ Length of the longest root $(\mathrm{cm})$} \\
\hline Season & \multicolumn{2}{|c|}{14 DAS } & \multicolumn{2}{|c|}{ 28DAS } & \multicolumn{2}{|c|}{35 DAS } & \multicolumn{2}{|c|}{ PI } & \multicolumn{2}{|c|}{$50 \% \mathrm{H}$} \\
\hline DS & \multicolumn{2}{|c|}{$5.5^{\mathrm{b}}$} & \multicolumn{2}{|c|}{$8.5^{b}$} & \multicolumn{2}{|c|}{$9.3^{b}$} & \multicolumn{2}{|c|}{$13.4^{\mathrm{b}}$} & \multicolumn{2}{|c|}{$17.0^{\mathrm{a}}$} \\
\hline WS & \multicolumn{2}{|c|}{$6.8^{\mathrm{a}}$} & \multicolumn{2}{|c|}{$11.0^{\mathrm{a}}$} & \multicolumn{2}{|c|}{$12.2^{\mathrm{a}}$} & \multicolumn{2}{|c|}{$14.4^{\mathrm{a}}$} & \multicolumn{2}{|c|}{$13.3^{\mathrm{b}}$} \\
\hline $\mathrm{CV} \%$ & \multicolumn{2}{|c|}{12.8} & \multicolumn{2}{|c|}{12.1} & \multicolumn{2}{|c|}{9.9} & \multicolumn{2}{|c|}{8.2} & \multicolumn{2}{|c|}{9.3} \\
\hline & \multicolumn{2}{|c|}{14 DAS } & \multicolumn{2}{|c|}{ 28DAS } & \multicolumn{2}{|c|}{35 DAS } & \multicolumn{2}{|c|}{ PI } & \multicolumn{2}{|c|}{$50 \% \mathrm{H}$} \\
\hline Trt. & DS & WS & DS & WS & DS & WS & DS & WS & DS & WS \\
\hline DDSR-UW & $4.6^{\mathrm{c}}$ & $6.4^{\mathrm{c}}$ & $8.7^{\mathrm{c}}$ & $6.4^{\mathrm{c}}$ & $9.1^{\mathrm{d}}$ & $9.1^{\mathrm{c}}$ & $13.2^{\mathrm{a}}$ & $15.2^{\mathrm{ab}}$ & $15.8^{\mathrm{d}}$ & $11.6^{\mathrm{c}}$ \\
\hline DDSR-RSM & $4.9^{\mathrm{c}}$ & $5.3^{d}$ & $8.7^{\mathrm{bc}}$ & $5.3^{d}$ & $8.9^{\mathrm{bc}}$ & $9.9^{\mathrm{c}}$ & $14.2^{\mathrm{a}}$ & $14.4^{\mathrm{bc}}$ & $16.2^{\mathrm{d}}$ & $12.4^{\mathrm{bc}}$ \\
\hline DDSR-C & $4.7^{\mathrm{c}}$ & $4.9^{\mathrm{d}}$ & $7.5^{\mathrm{d}}$ & $4.9^{\mathrm{d}}$ & $8.3^{\mathrm{cd}}$ & $7.9^{d}$ & $12.6^{\mathrm{b}}$ & $11.5^{\mathrm{d}}$ & $15.8^{\mathrm{cd}}$ & $12.8^{\mathrm{bc}}$ \\
\hline WDSR-UW & $5.5^{\mathrm{b}}$ & $7.4^{b}$ & $7.6^{\mathrm{bc}}$ & $7.4^{b}$ & $9.0^{\mathrm{ab}}$ & $11.6^{\mathrm{b}}$ & $14.2^{\mathrm{a}}$ & $16.0^{\mathrm{a}}$ & $18.0^{\mathrm{bc}}$ & $13.2^{\mathrm{bc}}$ \\
\hline WDSR-RSM & $7.2^{\mathrm{a}}$ & $8.5^{\mathrm{a}}$ & $10.5^{\mathrm{a}}$ & $8.5^{\mathrm{a}}$ & $11.0^{\mathrm{a}}$ & $14.6^{\mathrm{a}}$ & $14.2^{\mathrm{a}}$ & $15.5^{\mathrm{ab}}$ & $18.3^{\mathrm{a}}$ & $15.2^{\mathrm{a}}$ \\
\hline WDSR-C & $6.1^{\mathrm{b}}$ & $7.8^{\mathrm{ab}}$ & $8.1^{\mathrm{b}}$ & $7.8^{a b}$ & $9.6^{\mathrm{ab}}$ & $12.5^{\mathrm{b}}$ & $12.3^{b}$ & $13.2^{\mathrm{c}}$ & $18.0^{\mathrm{ab}}$ & $13.6^{\mathrm{b}}$ \\
\hline $\mathrm{CV} \%$ & 7.8 & 12.9 & 8.0 & 21.0 & 8.4 & 11.8 & 7.6 & 7.3 & 5.9 & 7.2 \\
\hline
\end{tabular}

DDSR, dry- direct seeded rice; WDSR, wet- direct seeded rice; UW, non-weeding; C, chemical; RSM, rice straw mulch; DS, dry season: WS, Wet season; DAS, days after sowing; Trt, treatment; PI, panicle initiation; H, heading In a column, values followed by a common letter are not significantly different at $\alpha=0.05$ 
Table 4. Total root length of rice per plant as affected by season, method of crop establishment and weed management

\begin{tabular}{|c|c|c|c|c|c|c|c|c|c|c|}
\hline \multicolumn{11}{|c|}{ Total root length /plant $(\mathrm{cm})$} \\
\hline Seas & \multicolumn{2}{|c|}{14 DAS } & \multicolumn{2}{|c|}{ 28DAS } & \multicolumn{2}{|c|}{$35 \mathrm{DAS}$} & \multicolumn{2}{|c|}{ PI } & \multicolumn{2}{|c|}{$50 \% \mathrm{H}$} \\
\hline DS & \multicolumn{2}{|c|}{$28.1^{\mathrm{a}}$} & \multicolumn{2}{|c|}{$83.3^{a}$} & \multicolumn{2}{|c|}{$102.4^{\mathrm{a}}$} & \multicolumn{2}{|c|}{$180.8^{b}$} & \multicolumn{2}{|c|}{$180.8^{b}$} \\
\hline WS & \multicolumn{2}{|c|}{$26.9^{\mathrm{a}}$} & \multicolumn{2}{|c|}{$84.4^{\mathrm{a}}$} & \multicolumn{2}{|c|}{$99.3^{\mathrm{a}}$} & \multicolumn{2}{|c|}{$205.8^{\mathrm{a}}$} & \multicolumn{2}{|c|}{$205.8^{\mathrm{a}}$} \\
\hline $\mathrm{CV} \%$ & \multirow{2}{*}{\multicolumn{2}{|c|}{$\frac{14.2}{14 \text { DAS }}$}} & \multirow{2}{*}{\multicolumn{2}{|c|}{$\frac{19.8}{28 \text { DAS }}$}} & \multicolumn{2}{|c|}{15.7} & \multicolumn{2}{|c|}{7.5} & \multicolumn{2}{|c|}{7.5} \\
\hline & & & & & & & & & & \\
\hline Trt. & DS & WS & DS & WS & DS & WS & DS & WS & DS & WS \\
\hline DDSR-UW & $19.1^{\mathrm{cd}}$ & $19.7^{\mathrm{c}}$ & $69.3^{\mathrm{c}}$ & $90.1^{\mathrm{b}}$ & $81.3^{\mathrm{c}}$ & $102.1^{\mathrm{bc}}$ & $153.7^{\mathrm{c}}$ & $199.5^{\mathrm{bcd}}$ & $142.52 \mathrm{c}$ & $199.7^{\mathrm{b}}$ \\
\hline DDSR-RSM & $21.2^{\mathrm{c}}$ & $9.9^{d}$ & $87.7^{\mathrm{b}}$ & $56.7^{\mathrm{c}}$ & $85.6^{\mathrm{c}}$ & $69.5^{\mathrm{d}}$ & $158.6^{\mathrm{c}}$ & $180.7^{\mathrm{d}}$ & $136.53 \mathrm{c}$ & $143.6^{\mathrm{c}}$ \\
\hline DDSR-C & $16.9^{\mathrm{d}}$ & $12.9^{\mathrm{d}}$ & $61.2^{\mathrm{d}}$ & $59.7^{\mathrm{c}}$ & $84.4^{\mathrm{c}}$ & $102.8^{\mathrm{bc}}$ & $161.2^{\mathrm{c}}$ & $183.0^{\mathrm{cd}}$ & $142.25 \mathrm{c}$ & $219.9^{\mathrm{a}}$ \\
\hline WDSR-UW & $33.4^{\mathrm{b}}$ & $34.4^{\mathrm{b}}$ & $71.2^{\mathrm{c}}$ & $81.9^{b}$ & $86.9^{\mathrm{c}}$ & $91.1^{\mathrm{c}}$ & $188.8^{\mathrm{b}}$ & $200.7^{\mathrm{bc}}$ & $192.72 \mathrm{~b}$ & $214.5^{\mathrm{ab}}$ \\
\hline WDSR-RSM & $46.3^{\mathrm{a}}$ & $48.5^{\mathrm{a}}$ & $132.9^{\mathrm{a}}$ & $113.3^{\mathrm{a}}$ & $164.6^{\mathrm{a}}$ & $120.9^{\mathrm{a}}$ & $235.6^{\mathrm{a}}$ & $264.2^{\mathrm{a}}$ & $228.29 \mathrm{a}$ & $225.8^{\mathrm{a}}$ \\
\hline WDSR-C & $31.5^{\mathrm{b}}$ & $33.4^{\mathrm{b}}$ & $77.5^{\mathrm{c}}$ & $100.7^{\mathrm{ab}}$ & $111.4^{\mathrm{b}}$ & $110.0^{\mathrm{ab}}$ & $187.0^{\mathrm{b}}$ & $203.2^{b}$ & $194.30 \mathrm{~b}$ & $207.5^{\mathrm{ab}}$ \\
\hline $\mathrm{CV} \%$ & 7.8 & 12.9 & 8.0 & 21.0 & 8.4 & 11.8 & 7.6 & 7.3 & 5.9 & 7.2 \\
\hline
\end{tabular}

DDSR, dry- direct seeded rice; WDSR, wet- direct seeded rice; UW, non-weeding; C, chemical; RSM, rice straw mulch; DS, dry season: WS, Wet season; DAS, days after sowing; Trt, treatment; PI, panicle initiation; $\mathrm{H}$, heading

In a column, values followed by a common letter are not significantly different at $\alpha=0.05$

\section{Shoot and root dry weights}

Shoot biomass was significantly influenced by the season at 28 DAS, panicle initiation and harvesting stages (Table 5). Although, a greater shoot biomass was produced until 50\% heading stage in the WS, at the time of harvesting a significantly higher shoot biomass was recorded in the DS. Among treatments, a significant increase in shoot biomass was recorded due to application of rice straw mulch in the WDSR method in both seasons. At initial stages, up to 35 days of sowing, the difference of the shoot biomass was influenced greatly by the rice straw mulch in WDSR. The rice shoots biomass produced in DDSR-RSM treatments was less when compared to WDSR-RSM in both seasons. A similar response to that of shoot biomass was observed in rice root biomass (Table 6). 
Table 5. Shoot dry weight of rice as affected by season, method of crop establishment and weed management

\begin{tabular}{|c|c|c|c|c|c|c|c|c|c|c|}
\hline \multicolumn{11}{|c|}{ Shoot dry weight $\left(\mathrm{g} / \mathrm{m}^{2}\right)$} \\
\hline Season & \multicolumn{2}{|c|}{28 DAS } & \multicolumn{2}{|c|}{35 DAS } & \multicolumn{2}{|c|}{ PI } & \multicolumn{2}{|c|}{$50 \% \mathrm{H}$} & \multicolumn{2}{|c|}{ Harv. } \\
\hline DS & \multicolumn{2}{|c|}{$44.9^{b}$} & \multicolumn{2}{|c|}{$79.3^{\mathrm{a}}$} & \multicolumn{2}{|c|}{$195.5^{\mathrm{b}}$} & \multicolumn{2}{|c|}{$496.0^{\mathrm{a}}$} & \multicolumn{2}{|c|}{$906.2^{\text {a }}$} \\
\hline WS & \multicolumn{2}{|c|}{$57.1^{\mathrm{a}}$} & \multicolumn{2}{|c|}{$91.6^{\mathrm{a}}$} & \multicolumn{2}{|c|}{$237.4^{\mathrm{a}}$} & \multicolumn{2}{|c|}{$469.3^{\mathrm{a}}$} & \multicolumn{2}{|c|}{$693.7^{\mathrm{b}}$} \\
\hline CV \% & \multicolumn{2}{|c|}{25.6} & \multicolumn{2}{|c|}{38.0} & \multicolumn{2}{|c|}{34.2} & \multicolumn{2}{|c|}{50.7} & \multicolumn{2}{|c|}{42.1} \\
\hline & \multicolumn{2}{|c|}{28 DAS } & \multicolumn{2}{|c|}{35 DAS } & \multicolumn{2}{|c|}{ PI } & \multicolumn{2}{|c|}{$50 \% \mathrm{H}$} & \multicolumn{2}{|c|}{ Harv. } \\
\hline & DS & WS & DS & WS & DS & WS & DS & WS & DS & WS \\
\hline DDSR-UW & $25.4^{\mathrm{c}}$ & $51.4^{\mathrm{c}}$ & $58.3^{\mathrm{b}}$ & $88.8^{\mathrm{b}}$ & $139.0^{\mathrm{b}}$ & $145.5^{b}$ & $266.6^{b}$ & $286.5^{\mathrm{c}}$ & $513.0^{\mathrm{c}}$ & $421.4^{b}$ \\
\hline DDSR-RSM & $27.8^{\mathrm{c}}$ & $44.5^{\mathrm{c}}$ & $47.1^{\mathrm{b}}$ & $107.6^{\mathrm{a}}$ & $164.8^{\mathrm{b}}$ & $194.0^{\mathrm{b}}$ & $499.7^{a b}$ & $461.3^{\mathrm{abc}}$ & $678.9^{\mathrm{bc}}$ & $613.4^{\mathrm{b}}$ \\
\hline DDSR-C & $30.3^{\mathrm{c}}$ & $34.7^{\mathrm{d}}$ & $63.0^{\mathrm{b}}$ & $92.4^{\mathrm{ab}}$ & $161.5^{b}$ & $278.3^{\mathrm{a}}$ & $564.6^{\mathrm{ab}}$ & $543.1^{\mathrm{ab}}$ & $867.2^{\mathrm{bc}}$ & $597.7^{b}$ \\
\hline WDSR-UW & $50.5^{b}$ & $44.8^{\mathrm{c}}$ & $64.4^{\mathrm{b}}$ & $85.7^{\mathrm{b}}$ & $162.9^{b}$ & $191.2^{b}$ & $327.9^{a b}$ & $286.5^{\mathrm{c}}$ & $774.7^{\mathrm{bc}}$ & $608.1^{b}$ \\
\hline WDSR-RSM & $95.3^{\mathrm{a}}$ & $94.1^{\mathrm{a}}$ & $157.7^{\mathrm{a}}$ & $95.7^{\mathrm{ab}}$ & $292.0^{\mathrm{a}}$ & $322.2^{\mathrm{a}}$ & $703.9^{\mathrm{a}}$ & $621.9^{\mathrm{a}}$ & $1145.5^{\mathrm{a}}$ & $987.5^{\mathrm{a}}$ \\
\hline WDSR-C & $39.9^{\mathrm{bc}}$ & $69.4^{\mathrm{b}}$ & $85.6^{\mathrm{b}}$ & $79.5^{\mathrm{b}}$ & $252.5^{\mathrm{ab}}$ & $300.0^{\mathrm{a}}$ & $613.6^{\mathrm{ab}}$ & $589.1^{\mathrm{a}}$ & $1458.3^{\mathrm{a}}$ & $918.5^{\mathrm{a}}$ \\
\hline CV \% & 32.7 & 11.6 & 43.4 & 15.1 & 48.7 & 20.6 & 62.2 & 62.2 & 47.1 & 31.8 \\
\hline
\end{tabular}

Table 6. Root dry weight or rice as affected by season, method of crop establishment and weed management

\begin{tabular}{|c|c|c|c|c|c|c|c|c|c|c|}
\hline \multicolumn{11}{|c|}{ Root dry weight $\left(\mathrm{g} / \mathrm{m}^{2}\right)$} \\
\hline 7 & \multicolumn{2}{|c|}{28 DAS } & \multicolumn{2}{|c|}{35 DAS } & \multicolumn{2}{|c|}{ PI } & \multicolumn{2}{|c|}{$50 \% \mathrm{H}$} & \multicolumn{2}{|c|}{ Harv. } \\
\hline DS & \multicolumn{2}{|c|}{$19.1^{\mathrm{b}}$} & \multicolumn{2}{|c|}{$33.8^{\mathrm{b}}$} & \multicolumn{2}{|c|}{$55.0^{\mathrm{b}}$} & \multicolumn{2}{|c|}{$61.6^{\mathrm{b}}$} & \multicolumn{2}{|c|}{$71.0^{\mathrm{a}}$} \\
\hline WS & \multicolumn{2}{|c|}{$27.6^{\mathrm{a}}$} & \multicolumn{2}{|c|}{$44.5^{\mathrm{a}}$} & \multicolumn{2}{|c|}{$68.1^{\mathrm{a}}$} & \multicolumn{2}{|c|}{$78.1^{\mathrm{a}}$} & \multicolumn{2}{|c|}{$63.3^{\mathrm{a}}$} \\
\hline $\mathrm{CV} \%$ & \multicolumn{2}{|c|}{24.3} & \multicolumn{2}{|c|}{21.9} & \multicolumn{2}{|c|}{25.0} & \multicolumn{2}{|c|}{46.7} & \multicolumn{2}{|c|}{29.9} \\
\hline & \multicolumn{2}{|c|}{28 DAS } & \multicolumn{2}{|c|}{35 DAS } & \multicolumn{2}{|c|}{ PI } & \multicolumn{2}{|c|}{$50 \% \mathrm{H}$} & \multicolumn{2}{|c|}{ Harv. } \\
\hline Trt. & DS & WS & DS & WS & DS & WS & DS & WS & DS & WS \\
\hline DDSR-UW & $10.4^{\mathrm{c}}$ & $12.1^{\mathrm{d}}$ & $19.3^{\mathrm{c}}$ & $31.8^{\text {de }}$ & $30.7^{\mathrm{b}}$ & $38.7^{\mathrm{c}}$ & $38.6^{\mathrm{b}}$ & $47.2^{\mathrm{b}}$ & $49.2^{\mathrm{c}}$ & $48.3^{b}$ \\
\hline DDSR-RSM & $12.6^{\mathrm{c}}$ & $20.2^{\mathrm{c}}$ & $24.2^{\mathrm{bc}}$ & $41.0^{\mathrm{c}}$ & $51.4^{\mathrm{b}}$ & $48.8^{c}$ & $44.1^{\mathrm{b}}$ & $44.1^{\mathrm{b}}$ & $76.7^{\mathrm{abc}}$ & $39.6^{b}$ \\
\hline DDSR-C & $13.5^{\mathrm{c}}$ & $15.5^{\mathrm{c}}$ & $31.0^{\mathrm{b}}$ & $28.6^{\mathrm{c}}$ & $42.5^{\mathrm{b}}$ & $51.1^{\mathrm{bc}}$ & $66.0^{\mathrm{b}}$ & $66.0^{\mathrm{b}}$ & $71.5^{\mathrm{abc}}$ & $56.9^{b}$ \\
\hline WDSR-UW & $15.4^{\mathrm{c}}$ & $36.1^{\mathrm{b}}$ & $33.0^{\mathrm{b}}$ & $55.8^{\mathrm{b}}$ & $51.9^{\mathrm{b}}$ & $63.7^{\mathrm{b}}$ & $51.4^{\mathrm{b}}$ & $51.4^{\mathrm{b}}$ & $57.2^{\mathrm{bc}}$ & $50.5^{\mathrm{b}}$ \\
\hline WDSR-RSM & $35.1^{\mathrm{a}}$ & $48.4^{\mathrm{a}}$ & $64.6^{\mathrm{a}}$ & $67.8^{\mathrm{a}}$ & $75.2^{\mathrm{a}}$ & $98.9^{\mathrm{a}}$ & $135.8^{\mathrm{a}}$ & $135.8^{\mathrm{a}}$ & $79.7^{\mathrm{ab}}$ & $87.8^{\mathrm{a}}$ \\
\hline WDSR-C & $27.7^{\mathrm{b}}$ & $34.7^{\mathrm{b}}$ & $33.1^{\mathrm{b}}$ & $39.2^{\mathrm{cd}}$ & $78.5^{\mathrm{a}}$ & $104.5^{\mathrm{a}}$ & $122.0^{\mathrm{a}}$ & $122.0^{\mathrm{a}}$ & $91.8^{\mathrm{a}}$ & $95.4^{\mathrm{a}}$ \\
\hline $\mathrm{CV} \%$ & 32.7 & 12.0 & 24.8 & 14.8 & 33.6 & 16.9 & 46.0 & 41.9 & 31.7 & 25.0 \\
\hline
\end{tabular}

DDSR, dry- direct seeded rice; WDSR, wet- direct seeded rice; UW, non-weeding; C, chemical; RSM, rice straw mulch; DS, dry season: WS, Wet season; DAS, days after sowing; Trt, treatment; DS, dry season; WS, wet season; PI, panicle initiation; $\mathrm{H}$, heading; Harv, harvesting

In a column, values followed by a common letter are not significantly different at $\alpha=0.05$

\section{Yield components}

Panicle numbers $/ \mathrm{m}^{2}$ (Table 7) were significantly affected by the season and treatments. In DS, rice plants had an average of 407 panicles per $\mathrm{m}^{2}$, which was $36 \%$ higher than in the WS $\left(262\right.$ panicles per $\mathrm{m}^{2}$ ). On the contrary, the mean number of spikelets per panicle in DS plants (62.9 spikelets per panicle) was $26 \%$ less than that in WS (85.4 spikelets per panicle). There was no significant difference in filled grain percentage between the two seasons. The 
treatment effect was not significant in filled grain percentage in the WS, whereas a significant low filled grain percentage was recorded in DDSR-UW in DS. The average 1000grain weights in WS were was $5 \%$ greater than in the DS. Although the treatment effect was not significant for 1000- grain weight in the WS, a significantly low filled grain percentage was recorded in DDSR-RSM in the DS (Table 7).

A significantly higher panicle number was produced in WDSR-RSM, WDSR-C and DDSR$\mathrm{C}$ treatments in both seasons. In both seasons, the panicle number per unit area was also significantly correlated with shoot biomass (Table 5) of the rice plant. The correlation coefficient values of $0.18,0.60,0.82,0.82$ and 0.83 respectively, at 28,35 , days after sowing, panicle initiation, $50 \%$ heading and harvesting was observed in DS. In contrast, the correlation coefficient values in WS were $0.47,0.86,0.77$ and 0.73 respectively for 28 after sowing, panicle initiation, $50 \%$ heading and harvesting. The correlation coefficient values reveal that the positive correlation between shoot biomass and panicle number is greater after panicle initiation of the rice plant.

Table 7. Yield components of rice as affected by the season, method of crop establishment and weed management

\begin{tabular}{|c|c|c|c|c|c|c|c|c|}
\hline & & \multicolumn{7}{|c|}{ Yield component of rice } \\
\hline & & \multicolumn{2}{|c|}{ Panicle no. $/ \mathrm{m}^{2}$} & \multicolumn{2}{|c|}{ Spikelet per panicle } & \multicolumn{2}{|c|}{ FG\% } & TGW \\
\hline \multicolumn{9}{|l|}{ Season } \\
\hline DS & \multicolumn{2}{|c|}{$407^{\mathrm{a}}$} & \multicolumn{2}{|c|}{$62.9^{b}$} & \multicolumn{2}{|c|}{$83.9^{\mathrm{a}}$} & \multicolumn{2}{|c|}{$20.5^{\mathrm{b}}$} \\
\hline WS & \multicolumn{2}{|c|}{$262^{\mathrm{b}}$} & \multicolumn{2}{|c|}{$85.4^{\mathrm{a}}$} & \multicolumn{2}{|c|}{$85.7^{\mathrm{a}}$} & \multicolumn{2}{|c|}{$21.6^{\mathrm{a}}$} \\
\hline $\mathrm{CV} \%$ & \multicolumn{2}{|c|}{30.71} & \multicolumn{2}{|c|}{15.70} & \multicolumn{2}{|c|}{5.67} & \multicolumn{2}{|c|}{7.18} \\
\hline \multirow[t]{2}{*}{ Treatment } & \multicolumn{2}{|c|}{ Panicle no. $/ \mathrm{m}^{2}$} & \multicolumn{2}{|c|}{ Spikelet per panicle } & \multicolumn{2}{|c|}{ FG $\%$} & \multicolumn{2}{|c|}{ TGW } \\
\hline & DS & WS & DS & WS & DS & WS & DS & WS \\
\hline DDSR-UW & $278.2^{b}$ & $164.5^{\mathrm{c}}$ & $44.1^{\mathrm{c}}$ & $77.6^{\mathrm{c}}$ & $81.2^{\mathrm{b}}$ & $82.9^{\mathrm{a}}$ & $19.8^{\mathrm{ab}}$ & $21.4^{\mathrm{a}}$ \\
\hline DDSR-RSM & $340.0^{\mathrm{ab}}$ & $228.5^{b}$ & $57.2^{\mathrm{bc}}$ & $81.0^{\mathrm{bc}}$ & $82.8^{\mathrm{ab}}$ & $83.8^{\mathrm{a}}$ & $19.2^{\mathrm{b}}$ & $22.1^{\mathrm{a}}$ \\
\hline DDSR-C & $494.9^{\mathrm{a}}$ & $318.6^{\mathrm{a}}$ & $65.2^{\mathrm{ab}}$ & $88.9^{\mathrm{b}}$ & $83.0^{\mathrm{ab}}$ & $85.9^{\mathrm{a}}$ & $20.1^{\mathrm{ab}}$ & $21.3^{\mathrm{a}}$ \\
\hline WDSR-UW & $355.7^{\mathrm{ab}}$ & $185.2^{\mathrm{bc}}$ & $47.2^{\mathrm{c}}$ & $78.2^{\mathrm{c}}$ & $83.6^{\mathrm{ab}}$ & $85.9^{\mathrm{a}}$ & $20.7^{\mathrm{ab}}$ & $21.3^{\mathrm{a}}$ \\
\hline WDSR-RSM & $500.1^{\mathrm{a}}$ & $327.6^{\mathrm{a}}$ & $76.0^{\mathrm{a}}$ & $100.4^{\mathrm{a}}$ & $88.1^{\mathrm{a}}$ & $88.4^{\mathrm{a}}$ & $21.2^{\mathrm{ab}}$ & $21.5^{\mathrm{a}}$ \\
\hline WDSR-C & $470.9^{\mathrm{a}}$ & $354.0^{\mathrm{a}}$ & $81.2^{\mathrm{a}}$ & $88.9^{b}$ & $85.0^{\mathrm{ab}}$ & $87.1^{\mathrm{a}}$ & $21.5^{\mathrm{a}}$ & $21.3^{\mathrm{a}}$ \\
\hline $\mathrm{CV} \%$ & 35.7 & 17.9 & 22.9 & 7.8 & 6.0 & 5.7 & 8.3 & 5.8 \\
\hline
\end{tabular}

DDSR, dry- direct seeded rice; WDSR, wet- direct seeded rice; UW, non-weeding; C, chemical; RSM, rice straw mulch; SPP, spikelets per panicle; FG, filled grain; TGW, 1000- grain weight; DS, dry season; WS, wet season In a column, values followed by a common letter are not significantly different at $\alpha=0.05$

\section{Grain yield}

Although the mean seasonal grain yield of rice (Table 8 ) in the dry season was greater when compared to that of the wet season, it was not statistically significant $(\mathrm{p}=0.26)$. Among the treatments, a significantly higher grain yield was produced in WDSR-C, WDSR-RSM and DDSR-C in both seasons. A minor rice yield gain of $0.83 \%$ in WS and a marginal yield loss of $1.8 \%$ were observed in DS in WDSR-RSM (Table 8) compared to WDSR-C. Hence, WDSR-RSM could produce a comparable yield as WDSR-C. In DDSR-C, the yield loss was $22.55 \%$ and $11.48 \%$ respectively for DS and WS when compared to WDSR-C. 
Table 8. Grain yield of rice as affected by season, method of crop establishment and weed management

\begin{tabular}{|c|c|c|c|c|}
\hline & \multicolumn{2}{|c|}{ Rice grain yield $t / h a$} & & \\
\hline \multicolumn{3}{|l|}{ Season } & \multirow{2}{*}{\multicolumn{2}{|c|}{$\begin{array}{c}\text { \% yield loss/gain compared to } \\
\text { WDSR - C }\end{array}$}} \\
\hline DS & \multicolumn{2}{|c|}{$4.65^{\mathrm{a}}$} & & \\
\hline WS & \multicolumn{2}{|c|}{$4.27^{\mathrm{a}}$} & & \\
\hline $\mathrm{CV} \%$ & \multicolumn{2}{|c|}{31.3} & & \\
\hline Treatment & DS & WS & DS & WS \\
\hline DDSR-UW & $2.71^{b}$ & $2.52^{b}$ & $-61.06 \%$ & $-58.07 \%$ \\
\hline DDSR-RSM & $3.06^{\mathrm{b}}$ & $3.42^{b}$ & $-56.03 \%$ & $-43.09 \%$ \\
\hline DDSR-C & $5.39^{\mathrm{a}}$ & $5.32^{\mathrm{a}}$ & $-22.55 \%$ & $-11.48 \%$ \\
\hline WDSR-UW & $2.96^{\mathrm{b}}$ & $2.47^{\mathrm{b}}$ & $-57.47 \%$ & $-58.90 \%$ \\
\hline WDSR-RSM & $6.83^{a}$ & $6.06^{\mathrm{a}}$ & $-1.80 \%$ & $+0.83 \%$ \\
\hline WDSR-C & $6.96^{\mathrm{a}}$ & $6.01^{\mathrm{a}}$ & - & - \\
\hline $\mathrm{CV} \%$ & 40.1 & 19.3 & & \\
\hline
\end{tabular}

\section{DISCUSSION}

In this study, establishment methods (dry- direct seeded and wet- direct seeded) along with weeding techniques had a significant impact on growth and yield of rice. The application of rice straw mulch at the rate of $4 \mathrm{t} / \mathrm{ha}$ affected the seedling density in the DDSR method in DS. The effect of a physical barrier, possible allelopathic potential of rice straw (Hassan et al., 1998) on germinating dry paddy seeds or effect of moisture status of soil in DDSR method could be the responsible factors to reduce the seedling density in DS with rice straw mulch. However, the same rice straw mulch did not affect the seedling density in WDSR method in both seasons.

Plant height is an imperative yield trait that is controlled by the genetic makeup of the plant, as well as growing conditions, seedling vigor and nutrient status (Sawar et al., 2001). The plant height, length of the longest root and total plant root length were increased by the rice straw mulch in WDSR when compared to the non-mulched plots and DDSR-RSM, especially at early stages. This may be due to weed control and additional supply of nutrients in rice straw treated WDSR plots, and thereby enhancing the vigor of rice plants. The enhancement of soil temperature (Devi Dayal et al., 1991) and moisture content in the upper portion of the soil due to the mulch may also provide an ideal environment for vigorous plant growth especially at initial stages of the rice crop.

In general, a healthy crop of a new improved rice variety, under optimum condition should bear about 500-600 panicles per $\mathrm{m}^{2}$ in the DS and 300-400 panicles per $\mathrm{m}^{2}$ in the WS (IRRI, 2009). The seedling numbers observed in the study had varying numbers of panicles. A higher number of panicles $/ \mathrm{m}^{2}$ was recorded in DDSR-C, WDSR-RSM and WDSR-C treatments; in which effective weed control was possible than in the non weeded and rice straw treated DDSR plots in both seasons. Therefore, the application of rice straw mulch in WDSR was effective to develop good seedling population and maintain of healthy panicle numbers as in the treatment which received the herbicide. 
Grain yields of rice vary depending on the cropping season in the tropics. The two distinct cropping seasons in the tropics are climatically differentiated by high solar radiation due to less precipitation and higher atmospheric temperature in dry seasons when compared to the wet seasons (Laza et al., 2003). Dry seasons have advantages over wet seasons in terms of yield performance of rice if adequate water is present. The maximum grain yield is $6 \mathrm{t} / \mathrm{ha}^{-1}$ for wet seasons (Yoshida, 1981) and $10 \mathrm{t} / \mathrm{ha}^{-1}$ for DS in the tropical irrigated rice systems under normal climatic conditions (Yoshida, 1981; Peng et al., 2000). Evans and De data (1979) described that high irradiance at any stage after panicle initiation was associated with high yield. This study, clearly shows that the shoot dry weight was increased in DS, after the heading stage than in the WS, and it may directly be related to the high irradiance in the DS which enhances the production of photosynthates and thereby carbohydrate accumulation into grains. Although, the yield enhancement was not significant in DS in this study, it was an eight percent yield increase in DS than in the WS. In addition, a high yield in rice straw treated WDSR could be due to suppression of the weeds over the initial 30-45 days which is considered the critical period for weed competition. In WS, mulch could be seen until panicle initiation (i.e. 45 days after sowing) and in DS, it was not observed after 30 days in WDSR-RSM plots.

\section{CONCLUSIONS}

The results of this study revealed that RSM could be applied in WDSR in both seasons and DDSR in WS at a rate of $4 \mathrm{t} / \mathrm{ha}$ without affecting the seedling density. In terms of plant growth parameters, the tallest plants, having an extensive root system were in the WDSRRSM method. The WDSR-RSM could produce a comparable yield as WDSR-C. The yield enhancements were due to two yield components - panicle numbers per unit area and numbers of spikelet per panicle. The DS favors a higher the number of panicles while in WS there were more spikelets per panicle. The common practice by a majority of the farmers in the study area is WDSR-C. However, this study revealed two alternative methods to this present method. One is the crop establishment method of WDSR along with rice straw mulch by saving the herbicide costs and reducing the harmful impacts to the environment. The other one is following the DDSR method with chemical weed control.

\section{ACKNOWLEDGEMENT}

The authors thank the Faculty of Agriculture, Rajarata University of Sri Lanka for providing the field and other facilities to conduct this research. The research was funded by University Grants Commission of Sri Lanka.

\section{REFERENCES}

Boyle, M., Frankenberger, W.T.Jr. and Stolzy, L.H. (1989). The influence of organic matter on soil aggregation and water infiltration. J. Prod. Agri. 2, 290-299.

Devi Dayal, Naik, P.R. and Dongre, B.N. (1991). Effect of mulching on soil temperature and groundnut yield during rabi-summer season. Groundnut News 3, 4.

DOA, (2001). Sri Lanka rice knowledge bank. [On line]. [Accessed on 02.02.2009].

Available at http://www.knowledgebank.irri.org/sriLanka/index.html. 
Evans, L.T. and De Datta, S.K. (1979). The relation between irradiance and grain yield of irrigated rice in the tropics, as influenced by cultivar, nitrogen fertilizer application, and month of planting. Field crop Res. 2, 1-17.

Hassan, S.M., Aidy, I.R., Bastawisi, A.O. and Draz, A.E. (1998). Weed management in rice using allelopathic rice varieties in Egypt. In: Olofsdotter, M. (Ed.) "Proceedings of Workshop on Allelopathy in Rice". pp. 27-37. 25-7 November, International Rice Research Institute, Manila, Philippines.

IRRI, (2009). International Rice Research Institute. Rice knowledge bank, Seeding rates and plant populations. [On line]. [Accessed on 08.04.5011]. Available at, http://www.knowledgebank.irri.org/landprep/plant-establishment-mainmenu-51/seedingrates-and-plant-populations-mainmenu-57.html.

Laza, R.C., Peng, S., Akita, S. and Saka, H. (2003). Contribution of biomass partitioning and translocation of grain yield under sub-optimum growing conditions in irrigated rice. Plant Prod. Sci. 6, 28-35.

Mandal, K.G., Misra, A.K., Hati , K.M., Bandyopadhyay, K.K., Ghosh, P.K. and Mohanty, M. (2004). Rice residue- management options and effects on soil properties and crop productivity. Food, Agriculture \& Environment Vol.2(1), 224-231. 2004.

Mendoza, T.C. and Samson, R. (1999). Strategies to avoid crop residue burning in the Philippine context. p. 13. International Conference of "Frostbite and Sun Burns" Canadian International Initiatives Toward Mitigating Climate Change hosted by International Program (IP) of the Canadia Environmental Network (CEN) and the Salvadorn Center for Appropriate Technology (CESTA) held on 24 April-May 2.

Peng, S., laza, R.M., Visperas, R.M. Sanico, A.L., Cassaman, K.G. and Khush, G.S. (2000). Grain yield of rice cultivars and lines developed in the Philippines since 1966. Crop Sci. 40, 125-133.

Sarwar, N., Maqsood, M., Wajid, S.A. and Anwar-ul-Haq, M. (2001). Impact of nursery seeding density, nitrogen, and seedling age on yield and yield attributes of fine rice. Chilean J. Agric. Res. vol.71 no.3 Chillán set. 2011.

Singh, R.B. (2000). Environmental consequences of agricultural development: a case study for the Green Revolution State of Haryana, India. Agr. Ecosys. Environ. 2000; 82, 97-103.

Singh, Y., Singh, B., and Timsina, J. (2005). Crop residue management for nutrient cycling and improving soil productivity in rice-based cropping systems in the tropics. Advances in agronomy. 85, 269-407.

Stewart, B.A. and Robinson, C.A. (1997). Are agro-ecosystems sustainable in semi-arid regions? Advances in agronomy. 60, 191-228.

Yoshida, S. (1981). Fundamentals of Rice Crop Science. Los Baños, Laguna, Philippines, International Rice Research Institute. 269 p. 